The Astrophysical Journal, 322:L5-L7, 1987 November 1

(1) 1987. The American Astronomical Society. All rights reserved. Printed in U.S.A.

\title{
A SEARCH FOR QSOs TO FIT A COSMOLOGICAL MODEL WITH FLAT, CLOSED SPATIAL SECTIONS
}

\author{
H. V. FAGUNDES AND U. F. WICHOSKI \\ Instituto de Física Teórica, Universidade Estadual Paulista, São Paulo, Brazil \\ Received 1987 June 15; accepted 1987 August 10
}

\begin{abstract}
A cosmological model with Einstein-de Sitter local metric and three-torus spatial topology predicts multiple images of cosmic sources like QSOs. Assuming that our Galaxy, in an early stage of its evolution, was such a source, we look in a quasar catalog for the ones that can possibly be interpreted as images of the Galaxy in that stage, expecting to fit a particular realization of that model.
\end{abstract}

Subject headings: cosmology - galaxies: The Galaxy - quasars

\section{INTRODUCTION}

The idea of Friedmann-Robertson-Walker (FRW) cosmological models with closed but nonspherical spatial sections (i.e., three-dimensional physical space) is not yet well known, but its literature is gradually increasing-see Ellis (1971), Sokolov and Shvartsman (1974), Gott (1980), Fang and Sato (1983), Zel'dovich and Novikov (1983), Fagundes (1985), Ellis and Schreiber (1986), and references therein. Our immediate motivation for such closed models is to avoid the infinite spaces of the open FRW models (Fagundes 1983), but they may also be seen as an alternative for explaining some puzzling questions, like the assumed spatial homogeneity of FRW models (Ellis and Schreiber 1986).

In this Letter we report an attempt to fit existing data on quasars to a model with Einstein-de Sitter metric and the simplest of the closed, flat spatial sections, which is the flat three-torus $T^{3}$. This space form can be thought of as the result of identifying opposite faces of an orthogonal parallelepiped-see, for example, Ellis (1971) or Sokolov and Shvartsman (1974).

Basically we assume that the active nucleus of our Galaxy is the remnant of a QSO (considering QSOs as living as long as approximately $10^{9} \mathrm{yr}$ ) with redshift beyond the galaxies' range, i.e., $Z \geq 1$. Then we search a QSO catalog for opposite pairs of such objects that might be interpreted as early images of the Galaxy, as predicted by $T^{3}$ cosmology-see Sokolov and Shvartsman (1974), or Fagundes (1986), for example. In this way the dimensions of the fundamental cell (the parallelepiped whose faces are pairwise identified) could be determined.

Note, however, that there are several possible topologies available for Einstein-de Sitter cosmology (Ellis 1971), and many possible fundamental cells for each of these topologies - e.g., the dimensions of our parallelepiped are in principle arbitrary, and its faces might not be perpendicular to each other. Besides, we do not even know whether the density ratio $\Omega$ is actually unity; only recently has astronomical research given a measure of support to this value (Loh and Spillar 1986). Here we adopt $\Omega=1$ both for simplicity and for being a popular belief among theoreticians.
Therefore, not much stock is to be put in our particular fits of the data to our model. We rather see the value of this work to be the detailing of a method for this kind of fit. A similar search has been made by Demianski and Lysik (1986), who looked for opposite, equidistant images of both galaxies and QSOs. We excluded galaxies because there are so many of them that it would be meaningless (at this stage), to interpret them as early images of the home Galaxy. We also excluded quasars with $Z<1$.

\section{THE SEARCH FOR OLD IMAGES OF THE GALAXY}

In Figure 1 we represent the fundamental cell, with our Galaxy at its center, $G=0$. This does not violate the Copernican principle, since the flat torus is homogeneous-cf. Ellis (1971). The identifications of faces preserve orientation: $\overrightarrow{\mathrm{ABCD}} \equiv \overrightarrow{\mathrm{IJKL}}, \overrightarrow{\mathrm{ABJI}} \equiv \overrightarrow{\mathrm{DCKL}}, \overrightarrow{\mathrm{ADLI}} \equiv \overrightarrow{\mathrm{BCKJ}}$. Points $\mathrm{G}_{1}$ through $G_{6}$ are the closest images of $G$ in the model, and their distances give the dimensions of the cell: $a=\mathrm{GG}_{1}=$ $\mathrm{GG}_{2}, b=\mathrm{GG}_{3}=\mathrm{GG}_{4}, c=\mathrm{GG}_{5}=\mathrm{GG}_{6}$.

The best QSO catalog available to us when this project was started was $A$ Revised Optical Catalog of Quasi-Stellar Objects (Hewitt and Burbidge 1980) and its Errata (Hewitt and Burbidge 1981). Later a few more recently discovered quasars were taken into consideration, making a total of about 1500 sources. In order to carry our search out by a computer program, we put these data on a diskette file, named QSOCAT. Both the data file and the search program were implemented in the UCSD Pascal language, on an Apple IIe-compatible microcomputer.

In looking for equidistant, oppositely lying QSOs, a discrepancy of up to $5 \%$ in the redshifts and up to $2^{\circ}$ deviation from exact opposition was allowed. These tolerances were needed so that we would obtain the desired result from the present data, but there may be physical reasons for them, like lens effect, local motion with respect to the comoving background, and errors in the determination of redshifts (see also Demianski and Lysik 1986). We found 32 pairs in the above conditions, or $0.0028 \%$ of the total number of pairs. They are listed in Table 1. 


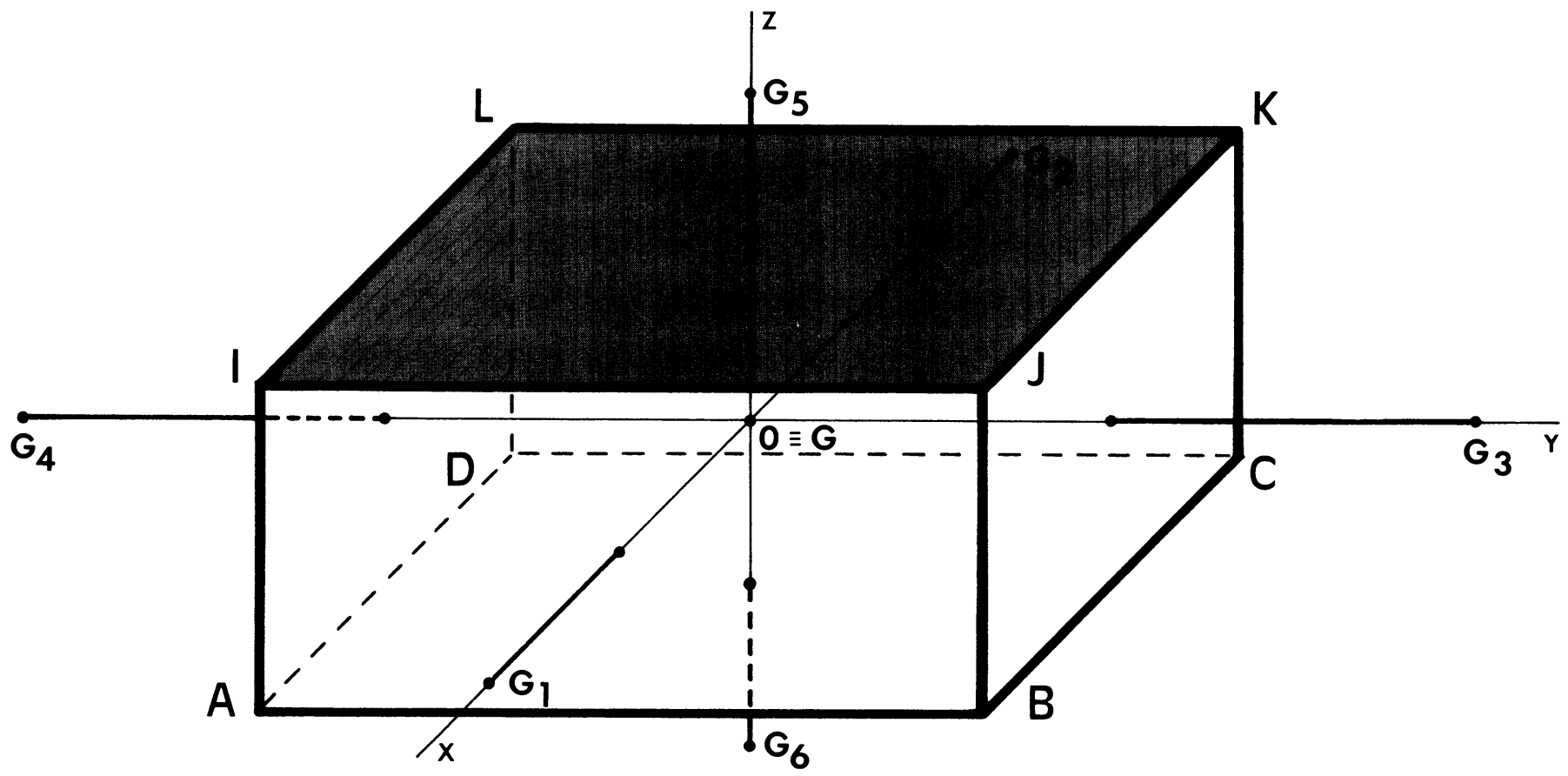

FIG. 1.- The orthogonal parallelepiped which is the fundamental cell in our model. The faces are pairwise identified: $\overrightarrow{\mathrm{ABCD}} \equiv \overrightarrow{\mathrm{IJKL}}, \overrightarrow{\mathrm{ABJI}} \equiv \overrightarrow{\mathrm{DCKL}}$, $\overrightarrow{\mathrm{ADLI}} \equiv \overrightarrow{\mathrm{BCKJ}}$. $\mathrm{G}$ is our Galaxy's position: $\mathrm{G}_{1}$ through $\mathrm{G}_{6}$ are the Galaxy's nearest images along the axes.

To see if this number reveals the correlation we are looking for, we tried a similar search in a simple Monte Carlo simulation of the uncorrelated problem, with 500 objects randomly distributed in space, up to $Z=2.5$. The average result of fifteen runs was 2.33 pairs, or $0.0019 \%$. But then it was noted that the higher percentage in the real search could be due to clustering (see, for example, rows 1 and 3 in Table 1 ). And that, by increasing the tolerance of $2^{\circ}$ from opposition to 2.3 in the simulation (to compensate for lack of clustering), an average result was 3.60 pairs, or $0.0029 \%$. So the question remains undecided and has to wait for more data and a better simulation.

Each of the pairs defines a potential axis for our fundamental cell, but because of the lack of exact alignment of its members with the Galaxy it was preferred to consider first each QSO individually as defining (with the Galaxy's position O) a potential semiaxis $\mathrm{O} x, \mathrm{O} y, \mathrm{O} z$. Then we searched for pairs of these semiaxes making an angle close to $90^{\circ}$, again with a tolerance of $2^{\circ}$. The results are presented in Table 2 , where the two cases correspond to the possible choices of semiaxes $\mathrm{O} x, \mathrm{O} y$ in the first half of the sky (R.A. $<12^{\mathrm{h}}$ ). In each case the choice was made so that the discrepancy between the redshifts of axis-defining QSOs was smallest, and/or the angle between the semiaxes was closest to $90^{\circ}$. See Table 3 . where the present distances of the images are also given, assuming a Hubble constant $H=75 \mathrm{~km} \mathrm{~s}^{-1} \mathrm{Mpc}^{-1}$. The distance $D$ for a redshift $Z$ is given by $D=(2 c / H)[1-(1$ $\left.+Z)^{-1 / 2}\right]=8000\left[1-(1+Z)^{-1 / 2}\right] \mathrm{Mpc}$.

From these choices we determined the $\mathrm{O} z$ direction to be R.A. $=13^{\mathrm{h}} .7$, decl. $=-65^{\circ} .7$ for case 1 ; and R.A. $=23^{\mathrm{h}} 7$, decl. $=48^{\circ} .9$ for case 2 . For case 1 we did not find any QSO along the $z$-axis. For case 2, just one was found, near the predicted $z$ direction: it was QSO $2332+489$, with $Z=1.534$, hence $D=2972 \mathrm{Mpc}$. Thus we get the cell dimensions as given in Table 4 , where for $x$-, $y$-directions we take the average distance of the oppositely lying QSOs as the measure of $a, b$.

From these values we can make predictions for the next nearest images on the $x, y$ plane, i.e., on points $( \pm a, \pm b, 0)$. But their redshifts are over 3.4, and we did not find any corresponding QSO to satisfy these conditions.

Returning to Table 4, it is interesting to note that the $x, y$ axes for both cases are in the same general direction on the sky. It could happen that these are not first images of the Galaxy in different fits, as we assumed, but rather the seventh and eighth images in each direction in a model like Ellis and Schreiber's (1986) small universes. We would then have $a \approx$ $455 \mathrm{Mpc}, b \approx 423 \mathrm{Mpc}$, and should look for the first six images in each direction among both QSOs and galaxies. But this possibility will not be pursued here.

\section{FINAL REMARKS}

We looked at the spectral lines of oppositely lying QSOs, but they seem too poorly known to warrant any reinforcement of the results. (If we allow the $x \mathrm{O} y$ angle to be $87^{\circ} .1$ we have a third case, with the $x, y$ axes defined by the pairs $[0151+048,1359-058]$ and $[2020-370,0812+367]$. The emission lines for each of these show a reasonable agreement.)

As stated in the Introduction, there are too many possible topologies and fundamental cells, and the above data could well be fitted to a number of them. (For sources other than the Galaxy, the search for early images is even more uncertain: one has to identify both the source and its repeated images, the latter no longer symmetrically located with re- 
TABLE 1

The 32 Pairs of APProximately OPPosite QSOS FOUND IN OUR SEARCH

\begin{tabular}{|c|c|c|c|c|c|}
\hline QSO & $Z^{\mathrm{a}}$ & $\mathrm{NC}^{\mathrm{b}}$ & $\begin{array}{l}\text { Opposite } \\
\text { QSO }\end{array}$ & $Z^{\mathrm{a}}$ & $\mathrm{NC}^{\mathrm{b}}$ \\
\hline $0002-387$ & 2.230 & 10 & $1207+398$ & 2.334 & 831 \\
\hline $0003-003$ & 1.037 & 21 & $1205-008$ & 1.002 & 828 \\
\hline $0014-392$. & 2.340 & 40 & $1207+398$ & 2.334 & 831 \\
\hline $0100-270$. & 1.597 & 132 & $1306+274$ & 1.537 & 938 \\
\hline $0102-389$ & 1.540 & 136 & $1255+372$ & 1.530 & 903 \\
\hline $0113-392$ & 2.070 & 155 & $1308+382$ & 2.090 & 944 \\
\hline \multirow[t]{2}{*}{$0117-380 \ldots \ldots$} & 2.020 & 160 & $1308+382$ & 2.090 & 944 \\
\hline & 2.020 & 160 & $1311+362$ & 2.084 & 951 \\
\hline $0117-400$. & 2.090 & 161 & $1308+382$ & 2.090 & 944 \\
\hline $0129-021$. & 1.390 & 187 & $1335+023$ & 1.356 & 979 \\
\hline $0130+038$ & 1.370 & 193 & $1328-034$ & 1.352 & 972 \\
\hline $0143+020$ & 1.600 & 244 & $1337-013$ & 1.607 & 983 \\
\hline $0151+048$ & 1.903 & 283 & $1359-058$ & 1.986 & 1007 \\
\hline $0402-362$ & 1.417 & 467 & $1611+343$ & 1.401 & 1133 \\
\hline \multirow[t]{4}{*}{$0407-199 \ldots \ldots$} & 1.986 & 472 & $1601+184$ & 1.940 & 1111 \\
\hline & 1.986 & 472 & $1604+181$ & 1.900 & 1117 \\
\hline & 1.986 & 472 & $1604+183$ & 2.000 & 1123 \\
\hline & 1.986 & 472 & $1605+179$ & 1.900 & 1125 \\
\hline \multirow{2}{*}{$0414-189$} & 1.536 & 474 & $1613+170$ & 1.520 & 1136 \\
\hline & 1.536 & 474 & $1613+173$ & 1.600 & 1140 \\
\hline $0438-166$ & 1.960 & 486 & $1634+176$ & 1.897 & 1162 \\
\hline $0812+367$ & 1.025 & 571 & $2020-370$ & 1.048 & 1229 \\
\hline $0848+155$ & 2.010 & 616 & $2044-168$ & 1.943 & 1238 \\
\hline $0848+163$ & 1.932 & 617 & $2044-168$ & 1.943 & 1238 \\
\hline $0921+347$ & 2.250 & 650 & $2116-358$ & 2.341 & 1254 \\
\hline $0945+114 \ldots$ & 1.760 & 668 & $2146-133$ & 1.800 & 1279 \\
\hline $1054-034$ & 2.100 & 742 & $2254+024$ & 2.090 & 1451 \\
\hline \multirow[t]{2}{*}{$1123+434 \ldots \ldots$} & 2.014 & 773 & $2315-424$ & 1.920 & 1478 \\
\hline & 2.014 & 773 & $2315-423$ & 2.020 & 1479 \\
\hline \multirow[t]{2}{*}{$1148-001$. } & 1.982 & 802 & $2341+010$ & 1.960 & 1518 \\
\hline & 1.982 & 802 & $2345+003$ & 1.960 & 1521 \\
\hline $1148-171$ & 1.751 & 803 & $2353+154$ & 1.801 & 1537 \\
\hline
\end{tabular}
$5 \%$.

${ }^{\text {a }}$ The emission redshifts $(Z)$ in each pair differ by at most ${ }^{\mathrm{b}} \mathrm{NC}$ is the number of order in the data file QSOCAT.

spect to us.) If cosmic space eventually reveals itself to be closed, we believe that the multiple images will be first recognized by other means, like emission lines or shape of the sources (Sokolov and Shvartsman 1974), and perhaps a better knowledge of galaxy and quasar evolution. Then the correct topology could be inferred. It would be an exquisite development to see the doubts about the value of $\Omega$ settled by the observation of repeated images of the Galaxy and other well-known objects and their clusters.
TABLE 2

\begin{tabular}{|c|c|c|c|c|}
\hline \multirow[b]{2}{*}{ Semiaxis } & \multicolumn{2}{|c|}{ QSO } & \multicolumn{2}{|c|}{ OPPOSITE QSO } \\
\hline & Name & $Z^{a}$ & Name & $Z^{\mathrm{a}}$ \\
\hline \multicolumn{5}{|c|}{ Case 1} \\
\hline$y$ & $\begin{array}{l}0407-199 \\
0945+114\end{array}$ & 1.986 & $\begin{array}{l}1601+184 \\
1604+181 \\
1604+183 \\
1605+179 \\
2146-133\end{array}$ & $\begin{array}{l}1.940 \\
1.900 \\
2.000 \\
1.900 \\
1.800\end{array}$ \\
\hline \multicolumn{5}{|c|}{ Case 2} \\
\hline & $\begin{array}{l}0414-189 \\
0921+347\end{array}$ & 1.536 & $\begin{array}{l}1613+170 \\
1613+173 \\
2116-358\end{array}$ & $\begin{array}{l}1.520 \\
1.600 \\
2.341\end{array}$ \\
\hline & $0921+347$ & 2.250 & $2110-358$ & 2.341 \\
\hline
\end{tabular}

${ }^{\mathrm{a}} Z$ is the emission redshift.

TABLE 3

OUR FinAl ChOICE For AXES $x, y^{\mathrm{a}}$

\begin{tabular}{cccccc}
\hline \hline & QSO IN O $x$ Direction & & QSO In O $y$ Direction \\
\cline { 5 - 6 } \cline { 5 - 6 } CASE & Name & $\begin{array}{c}\text { Distance } \\
(\mathrm{Mpc})\end{array}$ & Name & $\begin{array}{c}\text { Distance } \\
(\mathrm{Mpc})\end{array}$ \\
\hline $1 \ldots \ldots$ & $2146-133$ & 3217 & $0407-199$ & 3368 \\
$2 \ldots \ldots$ & $2116-358$ & 3620 & $0414-189$ & 2974 \\
\hline
\end{tabular}

${ }^{\mathrm{a}}$ The $x \mathrm{O} y$ angles are 90.2 for case 1 and $90^{\circ} .1$ for case 2 .

TABLE 4

Dimensions of THE Fundamental Cell

\begin{tabular}{cccc}
\hline Case & $a(\mathrm{Mpc})$ & $b(\mathrm{Mpc})$ & $c^{\mathrm{a}}(\mathrm{Mpc})$ \\
\hline $1 \ldots \ldots$ & 3200 & 3350 & $\ldots$ \\
$2 \ldots \ldots$ & 3591 & 2966 & 2972 \\
\hline
\end{tabular}

${ }^{a} c$ is not determined for case 1.

We are grateful to Laerte Sodré, Jr. for a conversation which clarified to us some astronomical questions. This work was supported by the Brazilian Government agencies Financiadora de Estudos e Projetos and Conselho Nacional de Desenvolvimento Científico e Tecnológico.
Demianski, M., and Lysik, M. 1986, preprint.

Ellis, G. F. R 1971, Gen, Rel, Grav. 2, 7

Ellis, G. F. R., and Schreiber, G. 1986, Phys. Letters, A115, 97.

Fagundes, H. V. 1983, Phys. Rev. Letters, 51, 517. 1985, Ap. J., 291, 450

1986, in Proc. of the 4th Marcel Grossmann Meeting On General Relativity, ed. R. Ruffini, (Amsterdam: Elsevier Science Publisher), p. 1559.

Fang, L. Z., and Sato, H. 1983, Comm. Theoret. Phys. (China), 2, 1055.

\section{REFERENCES}

Gott, J. R., III. 1980, M.N.R.A.S., 193, 153.

Hewitt, A., and Burbidge, G. 1980, Ap. J. Suppl., 43, 57. 1981, Ap. J. Suppl., 46, 113.

Loh, E. D., and Spillar, E. J. 1986, Ap. J. (Letters), 307, L1. Sokolov, D. D., and Shvartsman, V. F. 1974, Soviet Phys.-JETP, 39. 196.

Zel'dovich, Y. B., and Novikov, I. D. 1983, The Structure and Evolution of the Universe, ed. G. Steigman (Chicago: University of Chicago Press), $\S 2.4 .11$.

Helio V. Fagundes and Ubirajara F. Wichoski: Instituto de Física Teórica, Universidade Estadual Paulista, Rua Pamplona, 145, 01405 São Paulo, SP, Brazil 